\title{
Detection and Classification of Emotional State Based on Speech Signal
}

\author{
Hiba Adreese Younis \\ Mrewan Mustafa \\ Rahma Raad \\ hibaadreese@uomosul.edu.iq \\ College of Computer Science and Mathematics \\ University of Mosul, Mosul, Iraq
}

Received on: 13/03/2018

Accepted on: 24/01/2019

\begin{abstract}
In this research, an algorithm was proposed to automatically classify the mood of the speaker by referring to his speech. Three moods were adopted in this study, namely joy, sadness and anger in order to distinguish between them.

The principle of the algorithm work includes the initial treatment of the signal of by removing the silence and then cut the signal to a number of sections length of each 512 sample, and then treatment by window (Hamming window) followed by the process of extracting the characteristics such as energy, the basic frequency, resonance frequencies of each section and for all speech signals that Were recorded, which included 30 signals of persons between 15 and 25 years of age in order to prepare the database for the three moods and to draw the characteristic curves and for each mood.

The selection of signals was done from training and testing set for detectingthe mood of these signals by performing the previous steps and then comparing the resulting curve with the previous curves using the correlation coefficient and the Euclidean distance.

The algorithm gave good results when these characteristics were adopted in the classification process and by about $75 \%$.

keywords: emotional states, harmonic product spectrum, linear predictive coding

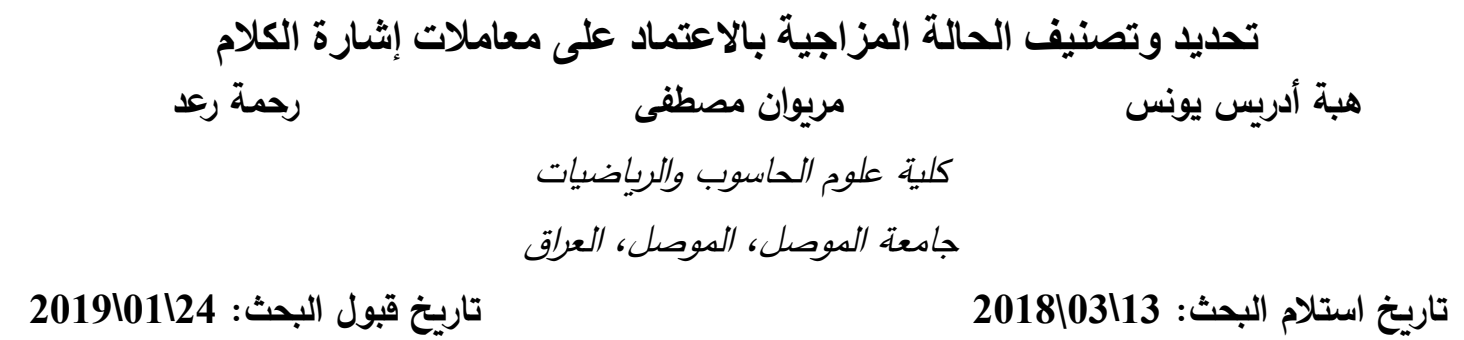

الملخص

اقترح في هذا البحث خوارزمية تعمل على التصنيف الآلي للحالة المزاجية للمتكلم بدلالة إثارة كلامه. إذ

اعتمد ثلاث حالات مزاجية في هذا البحث وهي الفرح والحزن والغضب من أجل التمييز بينها.

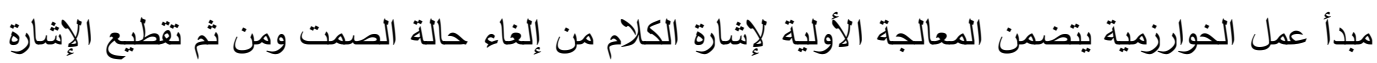

إلى عدد من المقاطع طول كل منها 512 عينة، ومن ثم المعالجة بالنافذة (Hamming Window) تليها عملية

استخلاص الخصائص المتمثلة بالطاقة، التردد الأساسي، والترددات الرنانة من كل مقطع ولجميع إثارات الكلام
\end{abstract}


التي سجلت والتي شملت30 إثارة كلام لأشخاص تتراوح أعمارهم بين 15و25 من الإناث، لأجل تهيئة قاعدة

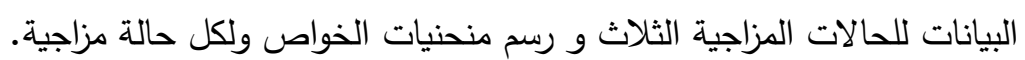
تتم عملية اختيار الإشارات من قاعدة البيانات من مجموعة التدريب والاختبار لتحديد حالتها المزاجية بإجراء الخطوات السابقة عليها ومن ثم مقارنة المنحني الناتج مع المنحنيات السابقة باستخدام مقاييس معامل الارتباط والمسافة الإقليدية.

وقد أعطت الخوارزمية نتائج جيدة عند اعتماد تلك الخصائص في عملية التصنيف وبنسبة 75\% تقريباً. الكلمات المفتاحية: الحالات المزاجية، خوارزمية ضرب توافقيات الأطياف، خوارزمية ترميز التتبؤ الخطي.

\section{1.المقدمة Introduction}

تلعب العاطفة دوراً مهماً في حياتنا اليومية الثخصية. وقد أظهرت الأبحاث السابقة أنه يمكن التعرف على

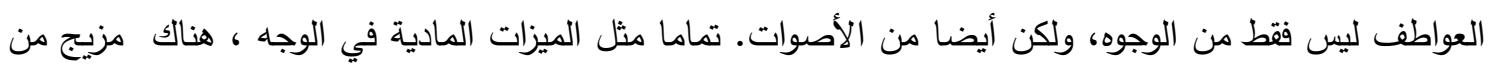

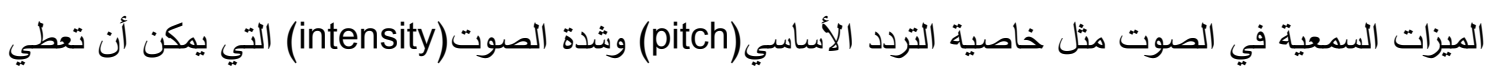
رؤية واضحة عن الحالات العاطفية الكامنة فيالفرد[1]. إن التمييز الآلي للحالة المزاجية من الكلام أصبح ذا أهمية متزايدة في السنوات الأخيرة في حقول ومجالات واسعة من التطبيقات التي استفادت من هذه التقانة[2].

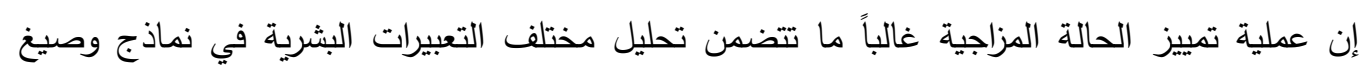
متعددة الوسائط مثل النصوص،أو الصوت، أو الفيديو. يكتثف أنواع مختلفة من الحالات المزاجيةبدمج المعلومات من تعابير الوجه وحركة الجسم والإيماءات والكلام[3]. إن العاطفة هي جزأ لا يتجزأ من قراراتنا اليومية العقلية والذكية، فهي ت تساعدناعلى التواصلمعبعضنا. البعض بالتعبير عن مشاعرنا. هذا الجانب الهام منالتفاعل البشري يحتاج إلى الأخذ بنظر الاعتبار تصنية تصديم

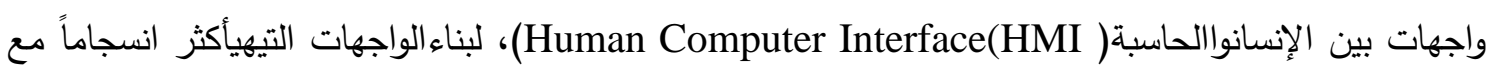

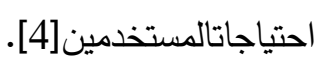
يمكن تصنيف الطرائق الحالية في التعرف على الحالة المزاجية لتصنيف أنواع معينة منها إلى ثلاث فئات

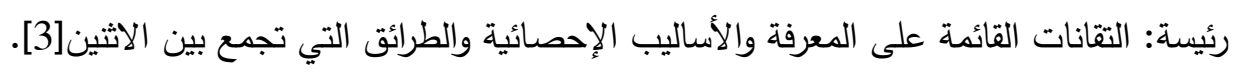

2. (اشارة الكلام (speech signal)

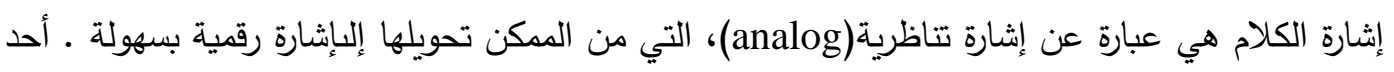
خصائص إثارة الكلام هي السعة التي من الممكن الحصول عليها بسهولة خلال إثارة الكلام الرقمية المسجلة.

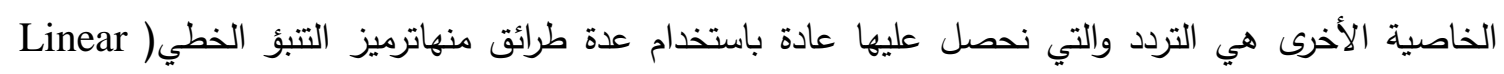
.(Predictive Coding إنإثارة الكلام التي ينتجها البشر تحوي معلومات أكثر من مجرد الرسالة التي يتم انتقالها فهي تعطي

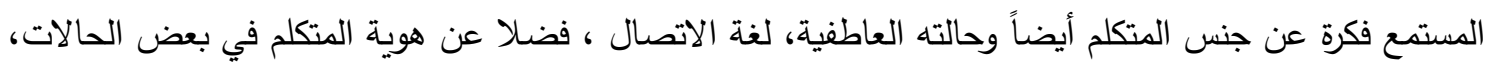

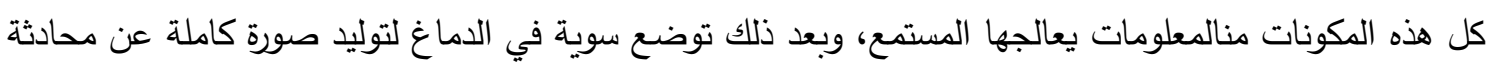


يمكن تصنيف المعلومات في إنـارة الكلام إلى ثلاثة مستويات:[5]

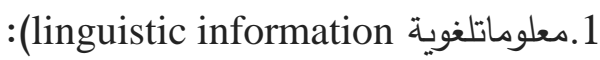
وتشملاكلمات(words)،المقاطع(syllables)،العبارات(phrases))التي يتكون منها الكلام. 2. معلومات لغوية إضافية (paralinguistis information): هذه المعلومات تمتلك مهيتين:

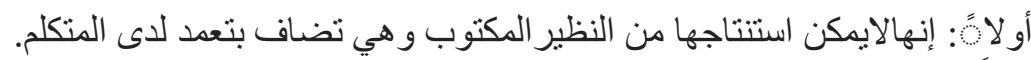

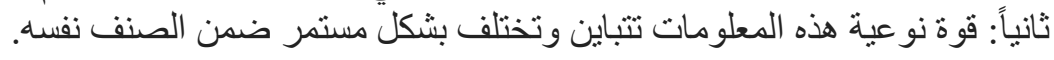

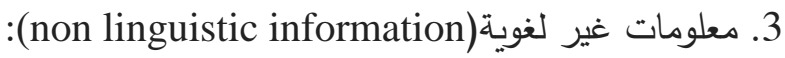

وهي المعلومـات التي لايمكنأن يضيفها المتكلم بتعمد مثل الجنس،العمر ،الحالـة الصـحية.هذه المعلومـات يمكن تصنيفها إلى: أولا: التغييرات الفردية المتعلقة بعلم وظائف الأعضاء وتثمل حجم ووزن وما يتعلق بعلم الأنسجة (عمر ) المنطقة الصوتية والتي تؤثر على الرنين في الكلام. ثانياً: ردود الأفعال التلقائية حسب الحالة العاطفية للمتكلم وتشمل الضحك، فئالبكاء،التثاؤب،التتفس السريع. 4.الدراسات السابقة ل قام الباحث سلر وآخرون(2002)، بتمييز العاطفة عن طريقة تحليل الكلام وحالة المستخدم لكلتا الحالتينبدراسة

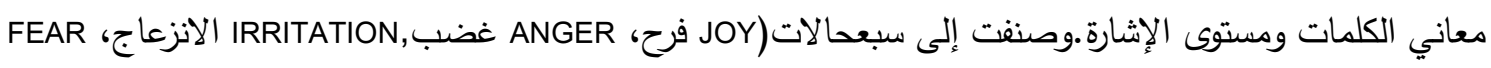

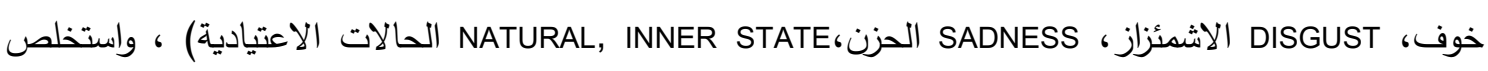

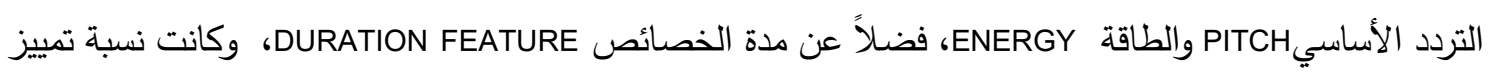

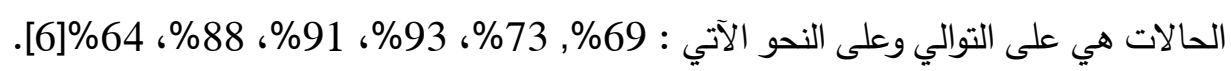

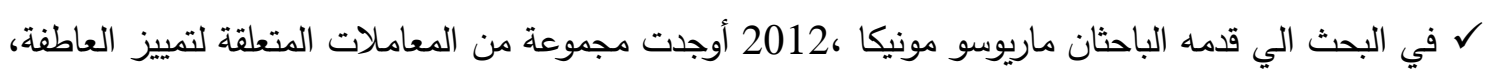

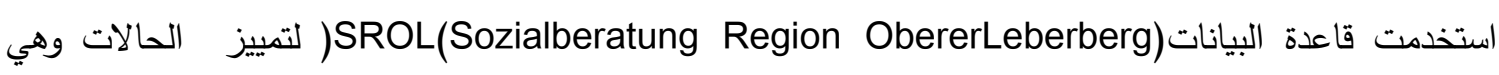
الفرح ، الخوف ، الحزن والحالات الاعتيادية، وكانت دقة التميز لتلك الحالات هي 63.97\% ، وتمت عملية التصنيف

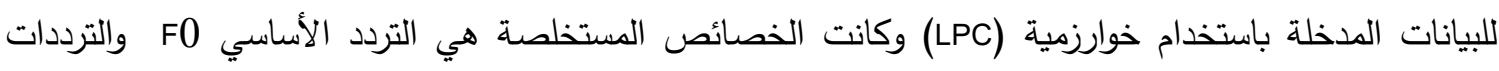

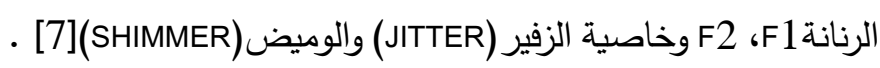
لو قام الباحثان (ميانك وتيم ،2012)بتحسين التعرف التلقائي للعاطفة من اشارة الكلام بدمج خصائص الإيقاع

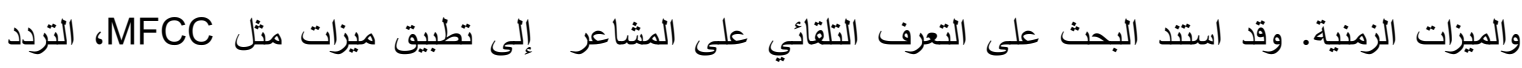

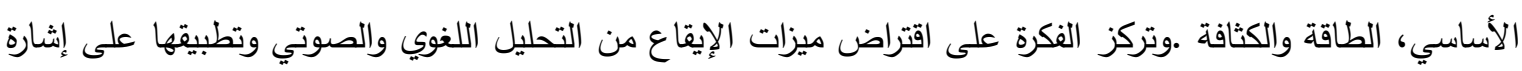
الكلام على أساس المعرفة الصوتية فقط .فضلاً عن الاستفادة من مجموعة من الميزات الزمنية والجهارة.

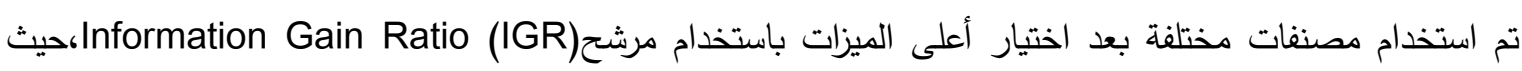

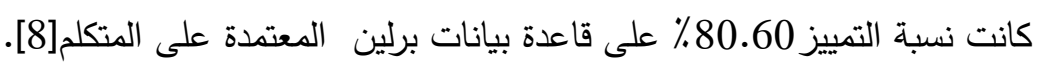

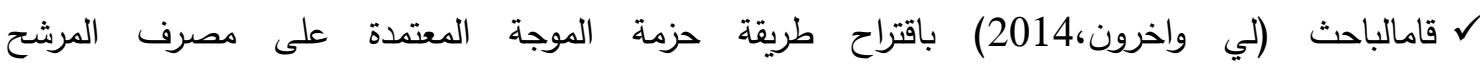
المحسنadaptive(filter bank، اذ استخلصت مجموعة من الخصائص السمعية مثل (CWPCC)، وقل وقد أثبتت النتائج أن Coiflet wavelet packet أكثر ملاعمة في تمييز الحالة المزاجية من باقي الحزم الموجية. كما أن الخصائص المقترحة حسنت عملية التمييز مقارنة بالخصائص القياسية[9]. 
ل قام الباحث (كلوغه وآخرون، 2017) باستخدام ماكنة التعلم الواسع(extreme learning machine) كمحلل آلي لعملية التمييز لإشارة الكلام، إذ طبقت على ثلاث قواعد بيانات مختلفة ، وقورنت النتائج التي تم الحصول عليها مع نتائج كل من support vector machine وطريقة deep learning المعتمد على تحليل التمييز العام وحسنت نسبة التمييز بمقدار 34-14\% مقارنة بطريقة support vector machine بينما حسنت نسبة

$$
\text { التمييز بمقدار 8\%-13\% مقارنة بطريقة تحليل التمييز العام[10]. }
$$

ل قام الباحث (كركيني وآخرون،2018) بمقارنة طرائق مختلفة لعملية تمييز الحالات المزاجية. إذ اقترح حل كفوء بالاعتماد على الدمج بين تلك الطرائق. استخدمت الثبكات العصبية ذات الارتداد العكسي) Recurrent Neural

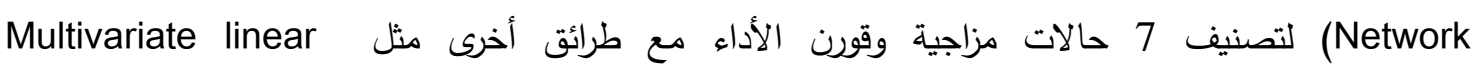

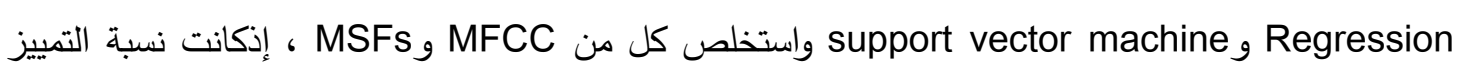

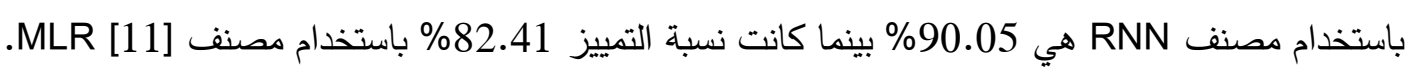

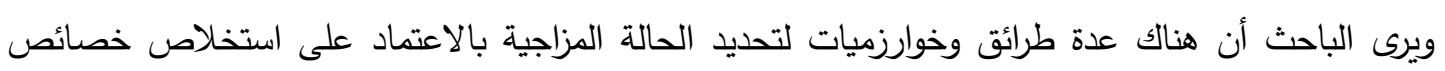

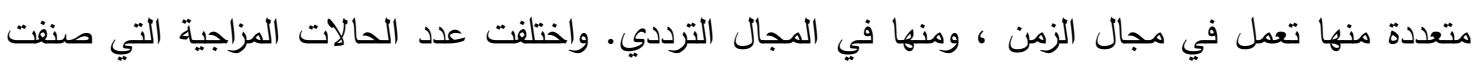

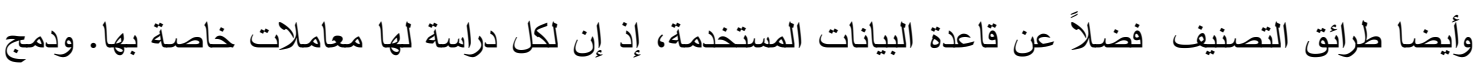
في هذا البحث بين الخصائص الزمنية والخصائص الترددية من أجل اعتمادها في المرحلة اللاحقة في عملية التصنيف التي أعطت نتائج جيدة بالاعتماد على قاعدة البيانات المكونة.

5.ستخلاص الخصائص

acoustic ) تمثل مرحلة تحليل الاثارة لاستخلاص الصفات الصوتية بدقة التي تمثل المعلومات الصوتية (information

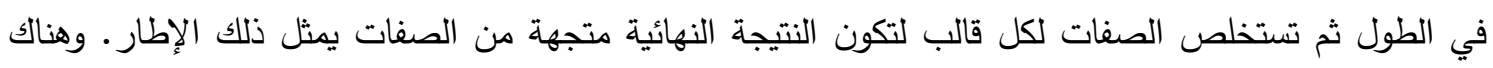

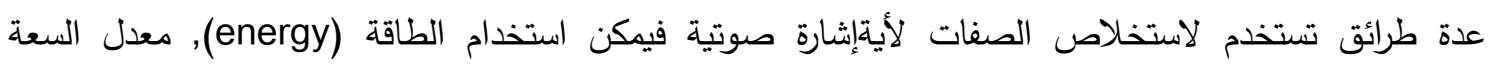
Linear (Lpc) (zerocrossing) معاملات التنبؤ الخطي (average magnetite) . [12](Predictive Coefficients 6 كوارزمية:خroduct spectrumharmonic ان التردد الأساسي يحوي معلومات عن المتكلم مثل الجنس، العمر، والحالة العاطفية. وهناك عدة طرائق لحساب قيمة التردد الأساسي، وبالاعتماد على طريقة الحساب يمكن تقسيمها إلى تحديد التردد الأساسي في مجال

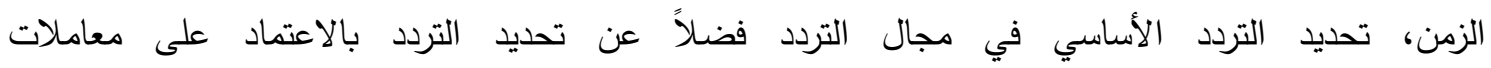

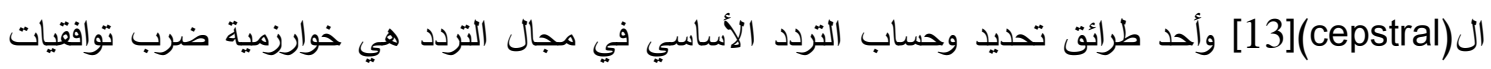

الأطياف(productspectrumharmonic)

في هذه الخوارزمية تقطع الإثارةإلى مقاطع segments بطول 512عينة، ولكل مقطع يطبق تحويل فورير

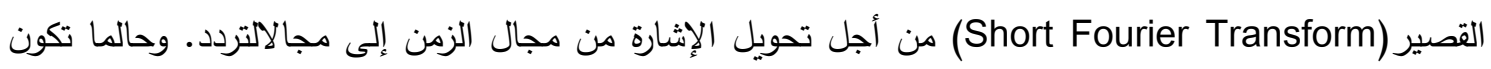
الإشارة في مجال التردد تطبق العمليتان الآتيتان لكل مقطع: 
• عملية الاختزال(downsampling): ولإجراء هذه العملية نقوم بكبس الطيف لكل مقطعبمقدار مرتين تارة

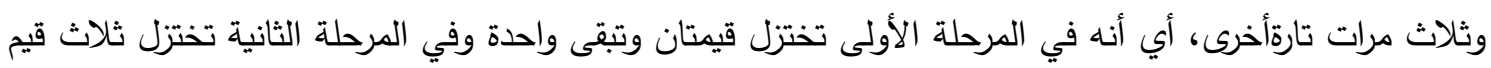
وتبقى واحدة. • عملية الضرب: وتتضمن عملية ضرب الأطياف الثلاثة سوية ومن ثم إيجاد الترددالذي يقابل أعلى قيمة(peak)

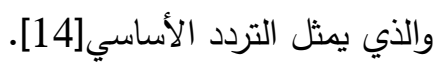
محاسن الخوارزمية

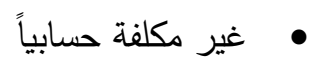
ملائمة لمختلف أنواعالإدخال ممكن نغير عدد الاطياف المكبوسة ممكن إحلال عملية جمع الأطياف بدل ضرب الإب الأطياف

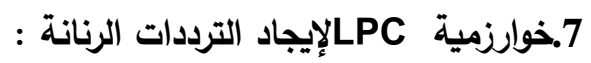

تعرف الترددات الرنانة بأنها القمم الطيفية في طيف الصوت. وتثير الترددات الرنانة في علم الكلام والصوتيات الى الرنين الصوتي للقناة الصوتية للشخص، إذ تمثل قمم السعة للطيف الترددي لموجة الصوت. لتونيك.

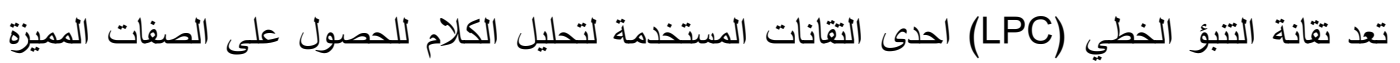

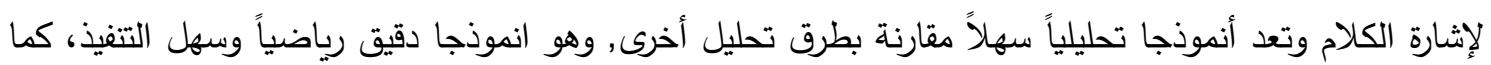

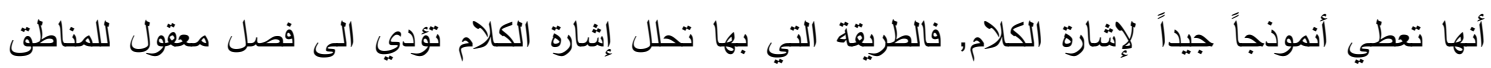
الصوتية للكلام على الرغم من أن هناك طرقاً عديدة لمعالجة الإثارة من البداية إلى النهاية إلا أبن ترميز التتبؤ الخطي أثبت نفسه وذلك بتحقيقه لنتائج جيدة بهذا المجال وهي طريقة رياضية دقيقة و بسيطة ويستطاع استخدامها

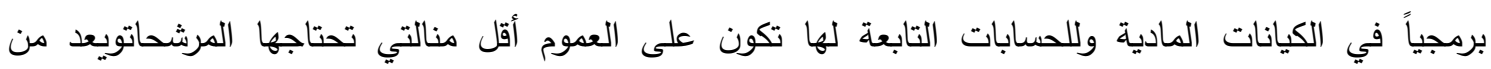

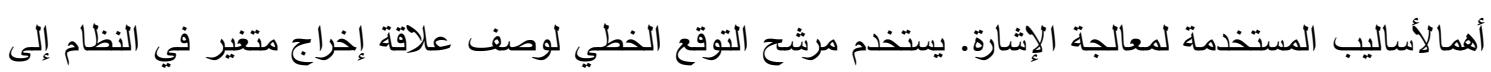
واحد أوأكثر من الإدخالات التي تعد محددات لوصفالنظام والتتي تحكم ببعادلات تفاضلية اعتيادية. إن طريقة ترميز التتبؤ الخطي المتغير مع الزمن والذي يمثل المنطقة الصوتية تستخدم خوارزمية LLPC

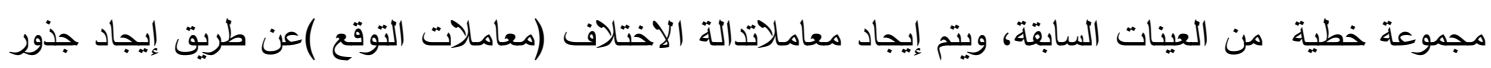
متعددة الحدود والتي تمثل الترددات الرنانة،إذأن كل زوج من الجذور المعقدة تستخدم لحساب الترددات الرنانة

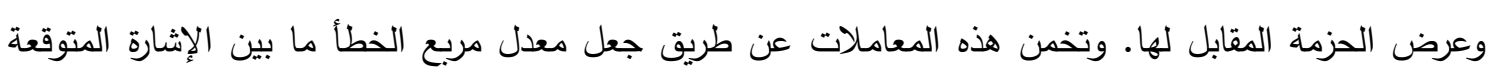
والحقيقية أقل ما يمكن[15].

8. الخوارزمية المقترحة لتصنيف الحالة المزاجية بالاعتماد على معاملات إثارة الكلام تتكون الخوارزمية المقترحة من مرحلتين رئيستين: المرحلة الأولى: مرحلة إعداد قاعدة البيانات تتكون هذه المرحلة من عملية تسجيل مجموعة من إشارات الكلام العشوائية لأصوات المتكلمين(الإناث) تتراوح أعمارهم بين(15-25) سنة، إذ تمت عملية التسجيل في غرفة ذات ظروف ملائمة (نسبة الضوضاء والصدى ملئ 
قليلة) باستخدام جهاز الموبايل النقال ذات المواصفات والدقة العالية استخدمت الإعدادات التالية في عملية التسجيل الصوتي:

$$
\text { تردد التعيان: } 8000 \text { عينة/ثانية }
$$

double:نوع البيانات المستخدمة للتسجيل:

مدة التسجيل: 3 ثانية

مادة التسجيل:

ل نجحت في الامتحان( في حالة الفرح)

ل

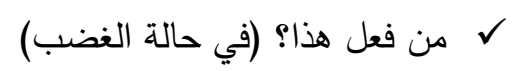

• • نوع التسجيل: أحادي (mono)

وقسمت في هذه المرحلة من الخوارزمية الإثارة بعد إلغاء حالة الصمت باستخدام برنامج(cool edit pro2)

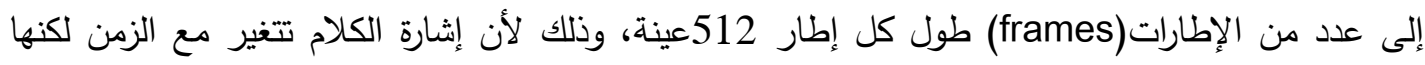

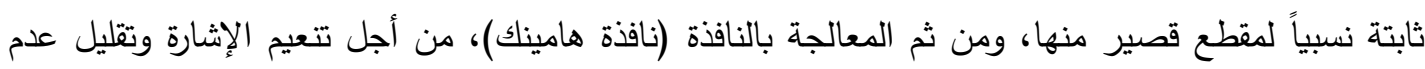

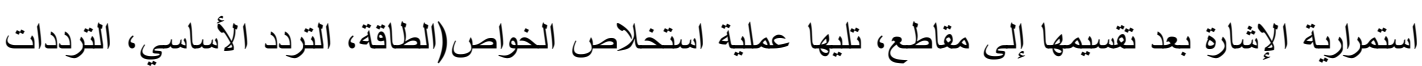

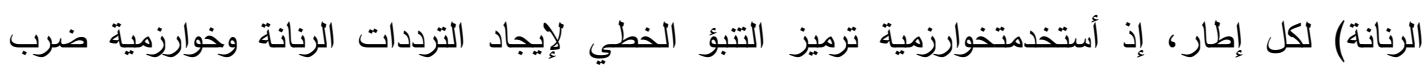

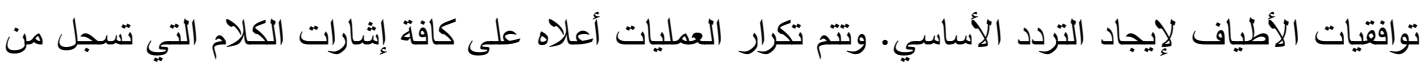

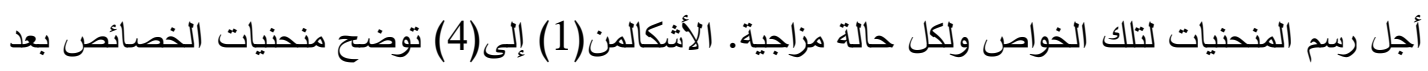

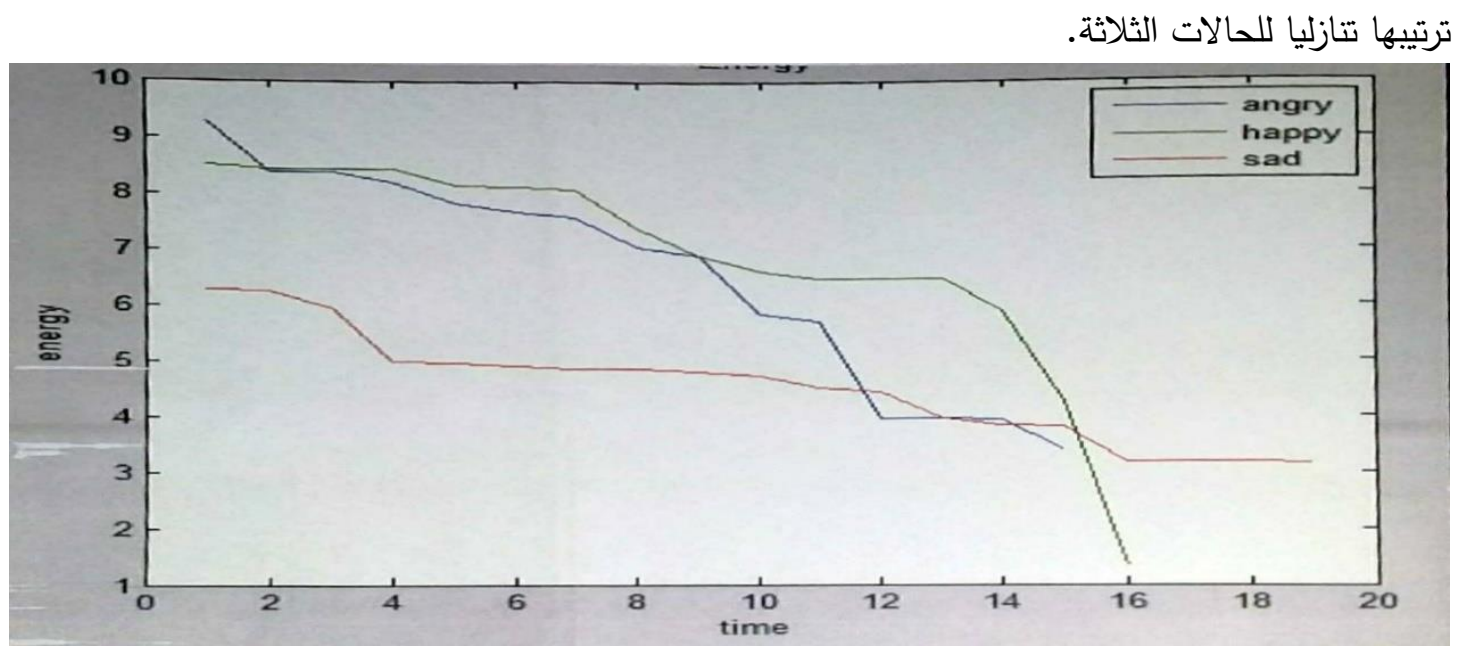

الثكل (1): قيمة الطاقة للحالات الثلاث 


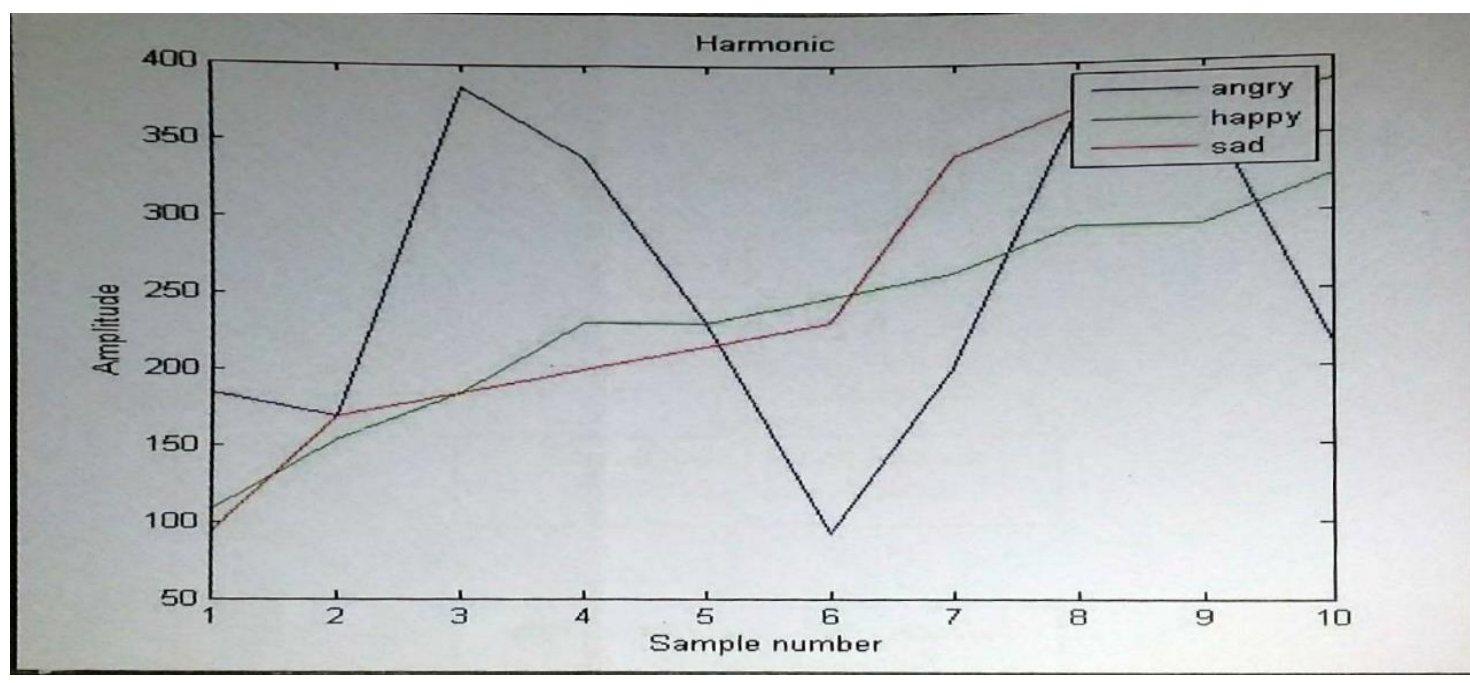

الثكل (2): قيمة التردد الأساسي للحالات الثلاث

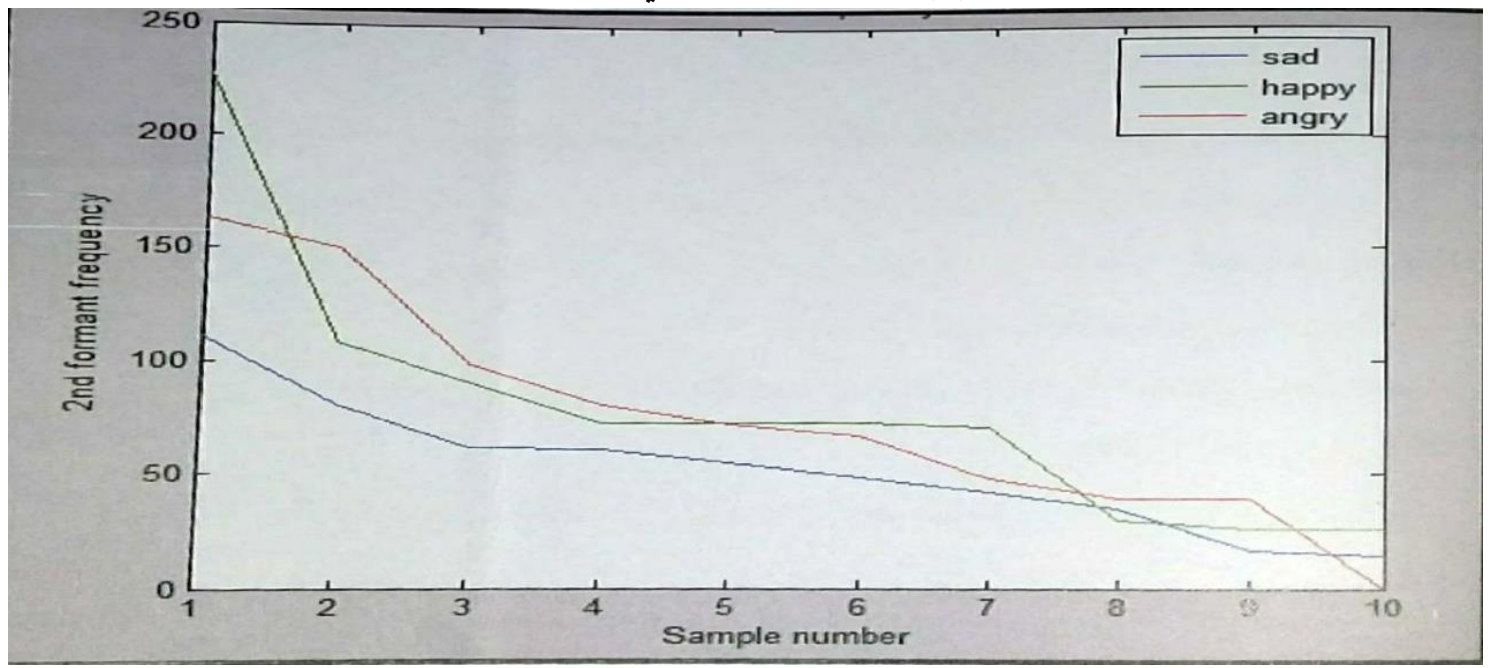

الثكل (3): قيمة التردد الرنان الثاني للحالات الثلاث

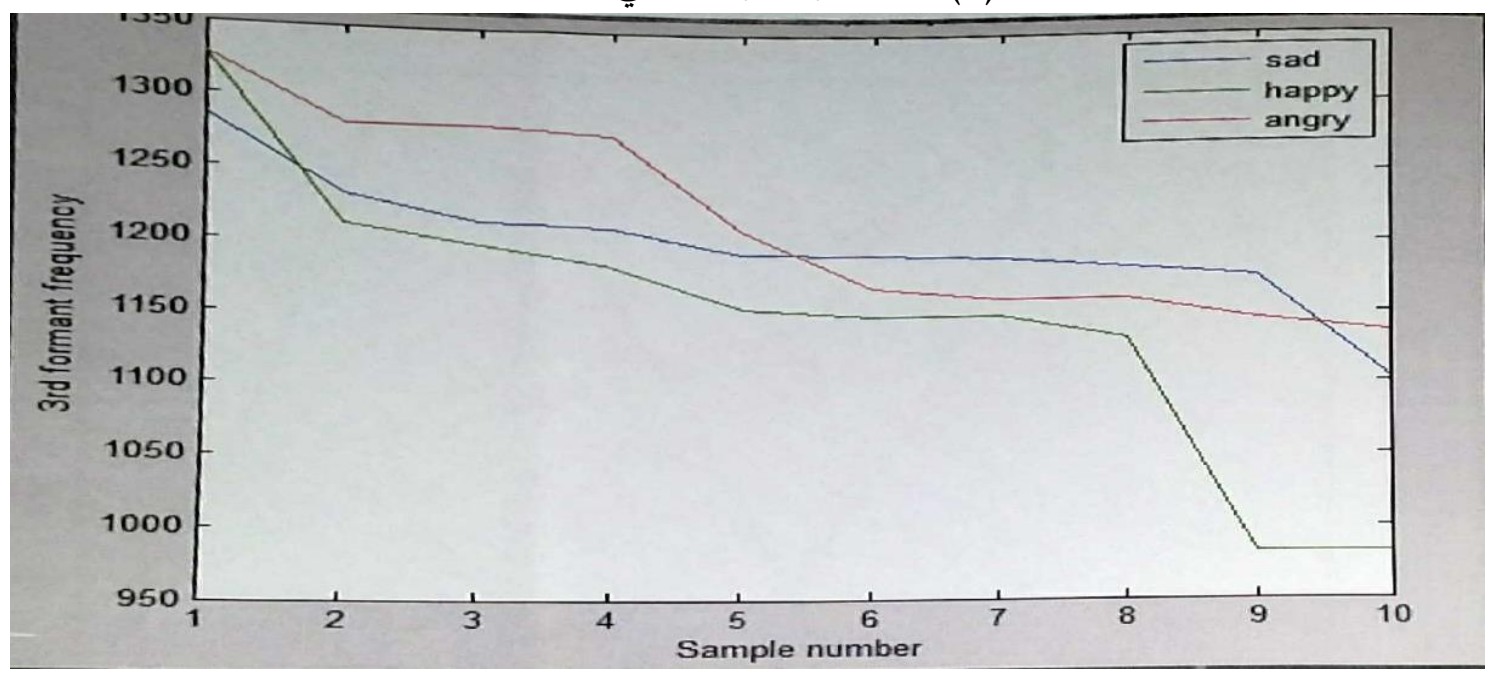

الثكل (4): قيمة التردد الرنان الثالث للحالات الثلاث

المرحلة الثانية: تحديد الحالة المزاجية للمتكلم 
يختار في هذه المرحلة عدد من إثارات الكلام العشوائية، قسم منها من داخل قاعدة البيانات والأخرى خارجية، إذ

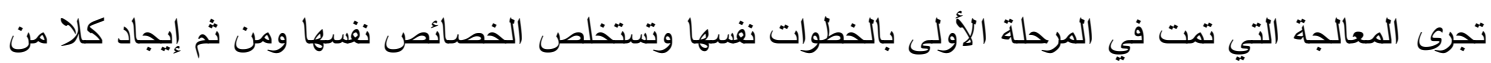

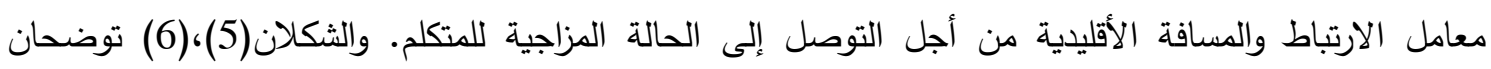
المخططان الصندوقين لمراحل الخوارزمية.

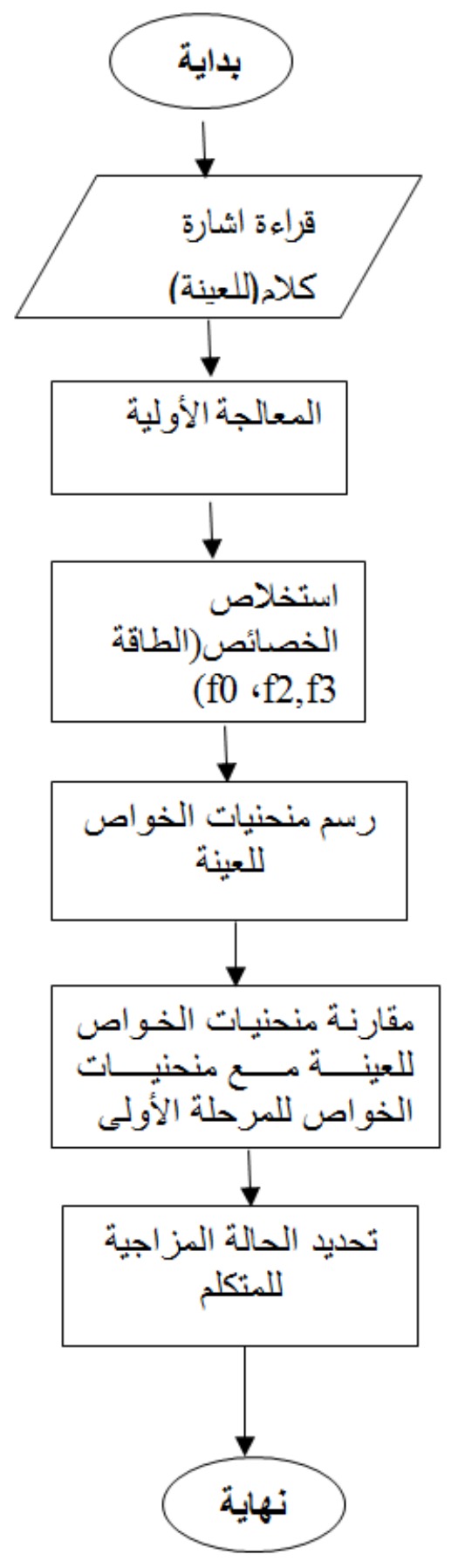

الثكل(6): المخطط الصندوقي للمرحلة الثانية (التصنيف)

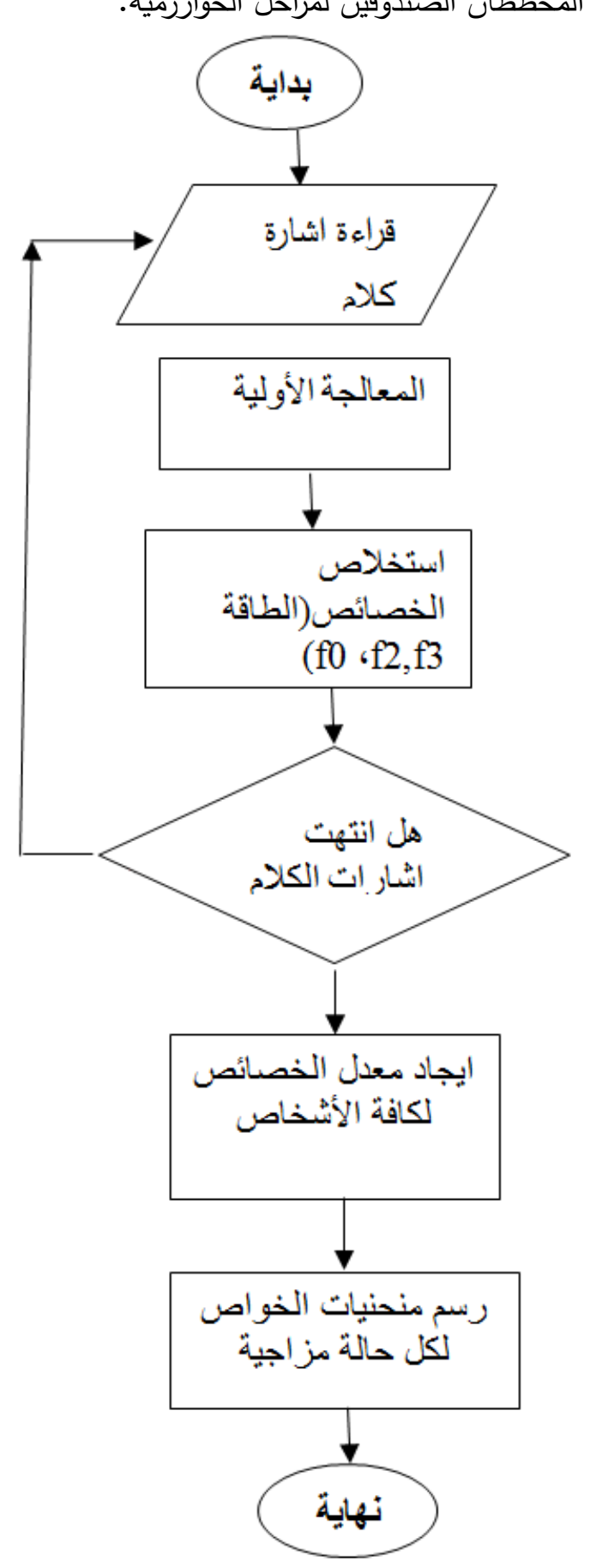

الشكل(5): المخطط الصندوقي للمرحلة الأولى (إعداد قاعدة البيانات) 
قرئ في هذا المثال ملف صوتي في حالة الفرح(happy.wav) وأجريت المعالجة الأولية عليه ومن ثم يتم تقطيع الإثارة إلى عدد من المقاطع طول كل مقطع 512عينة ليتم فيما بعد استخلاص الخواص منها والمتمثلة بالطاقة والتردد الأساسي والترددات الرنانة والأشكال من(7) إلى(10) تمثل منحنيات تلك الخواص.

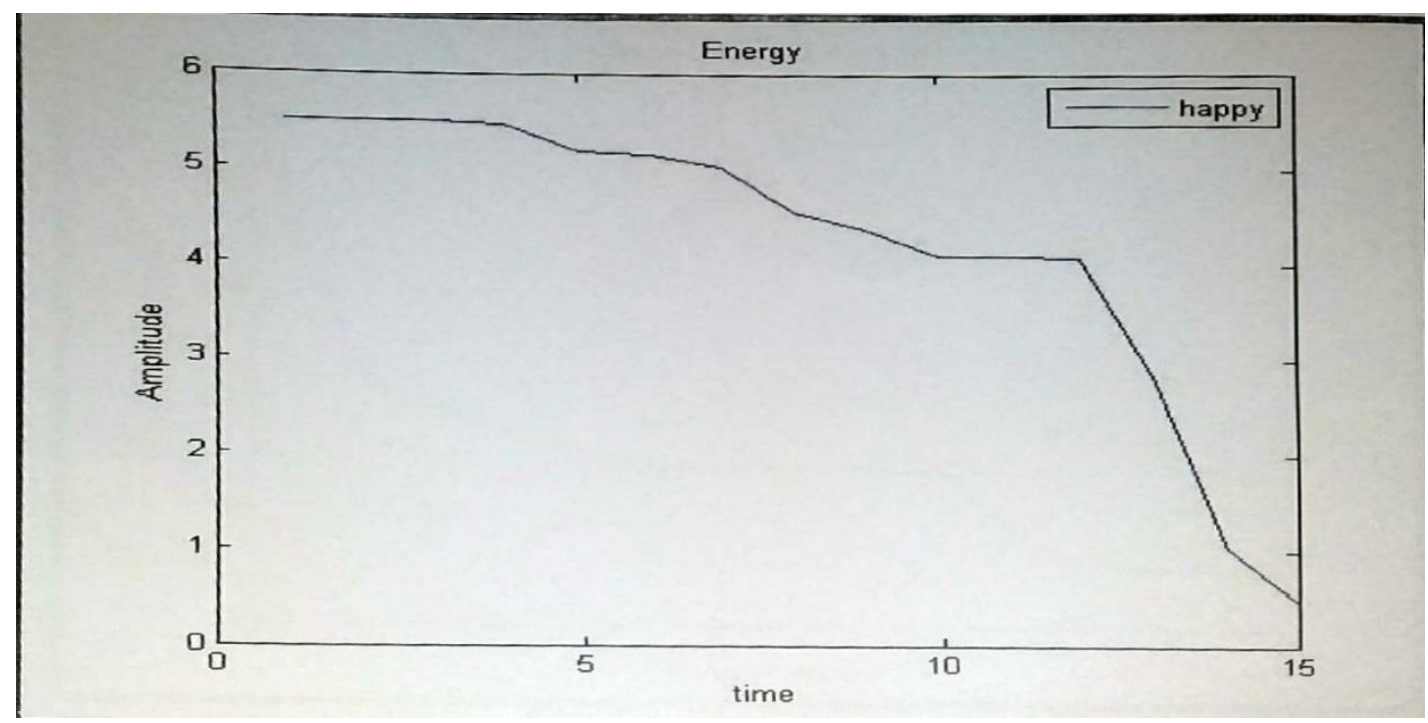

الشكل(7): قيمة الطاقة للعينة

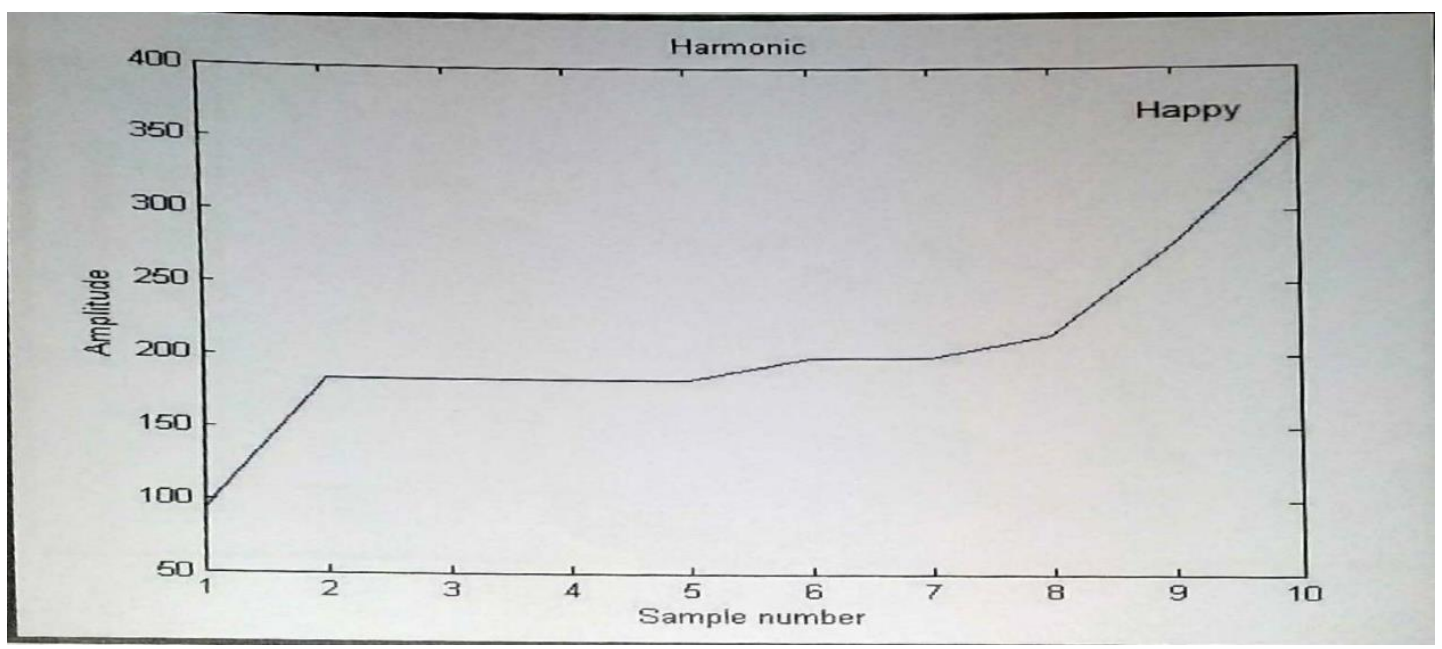

الثكل(8): قيمة التردد الأساسي للعينة 


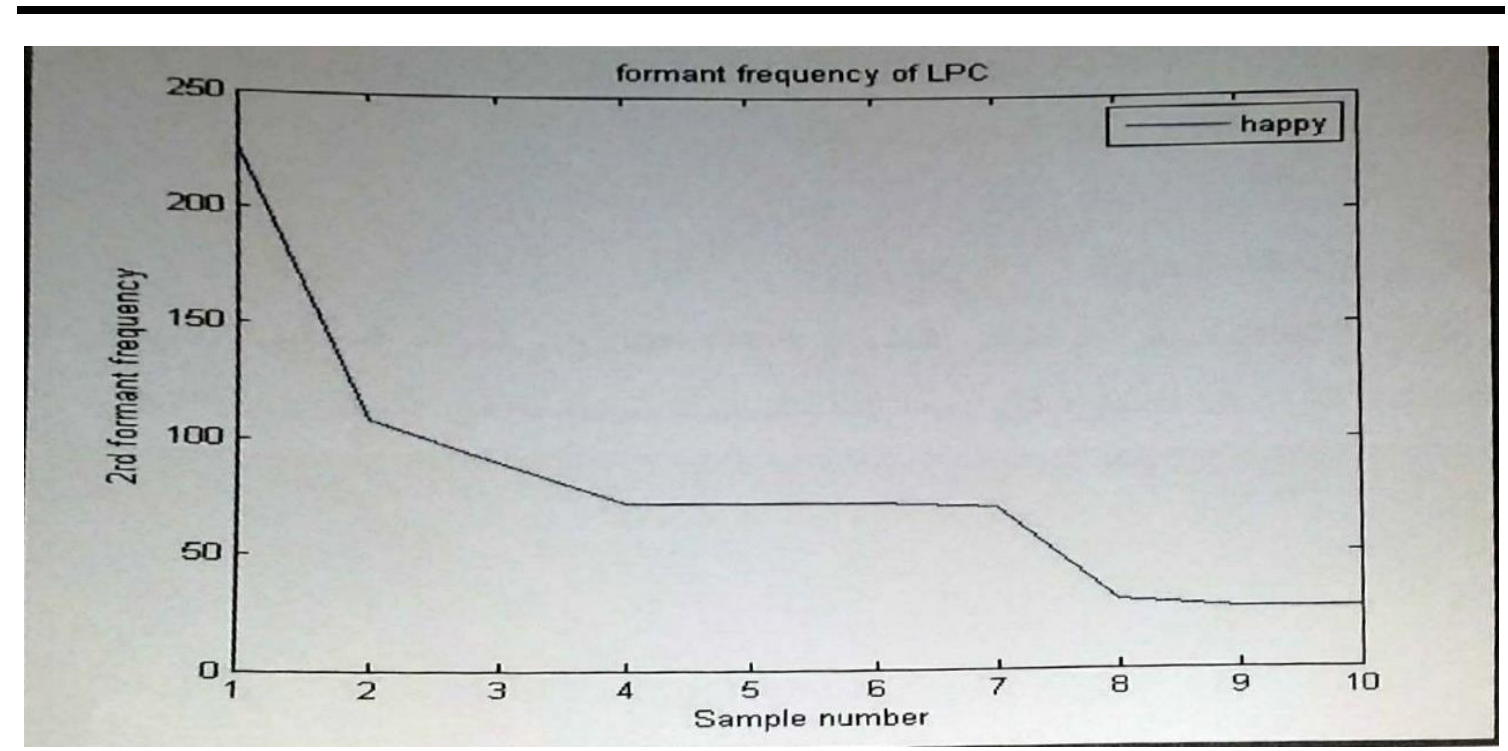

الثكل(9): قيمة التردد الرنان الثاني للعينة

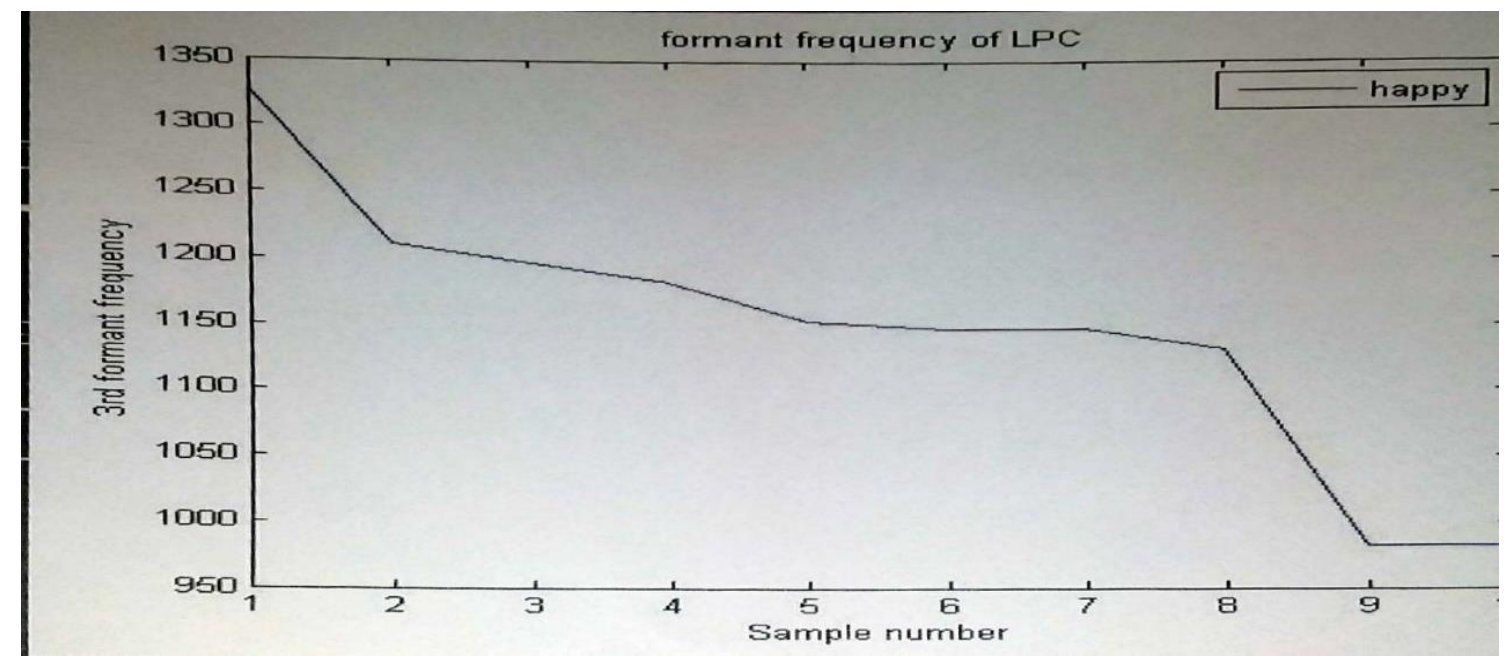

الثكل(10): قيمة التردد الرنان الثالث للعينة

وبعد إيجاد قيم تلك الخصائص للثخص المراد تحديد حالته المزاجية يتم إيجاد كل من معامل الارتباط والمسافة الإقليدية بين قيم تلك الخصائص والقيم المقابلة لها في المنحنيات السابقة التي تم الحصول عليها في مرحلة إعداد

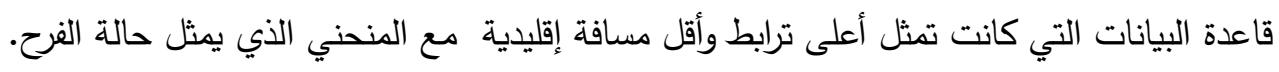

\section{• خ حالة الحزن}

قرئ في هذا المثال ملف صوتي في حالة الفرح(sad.wav) وأجريت المعالجة الأولية عليه ومن ثم تقطع الإشارة إلى عدد من المقاطع طول كل مقطع 512عينة ليتم فيما بعد استخلاص الخواص منها والمتمثلة بالطاقة والتردد الأساسي والترددات الرنانة والأشكال من(11) إلى (14) تمثل منحنيات تلك الخواص. 


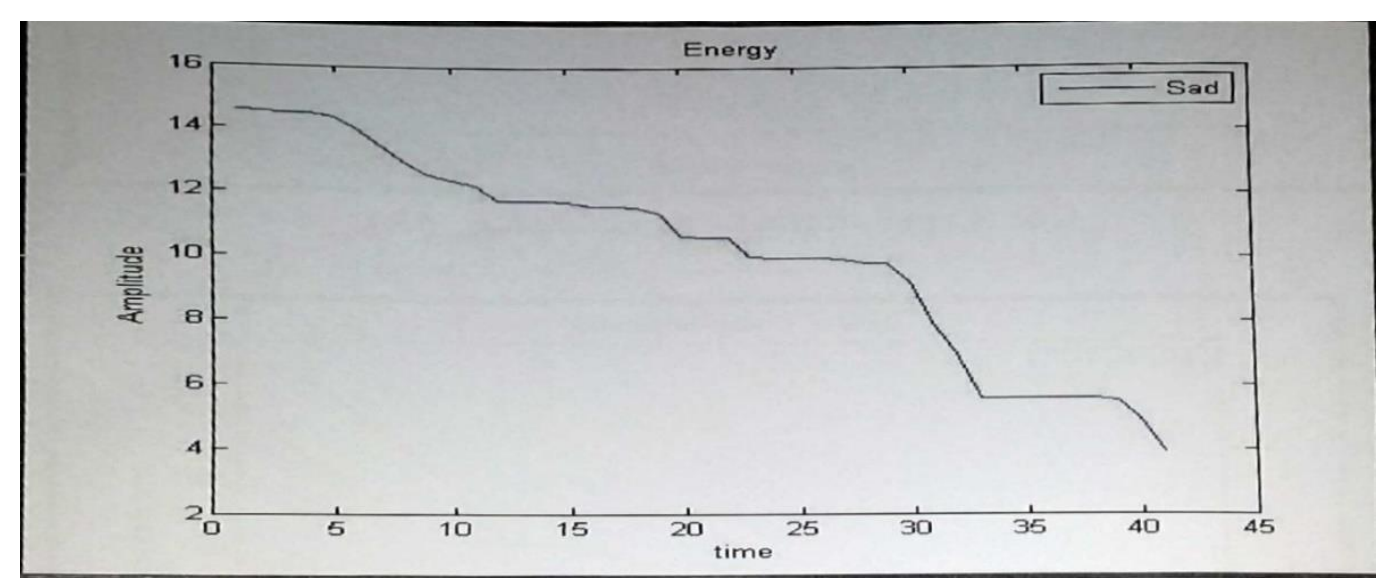

الشكل(11): قيمة الطاقة للعينة

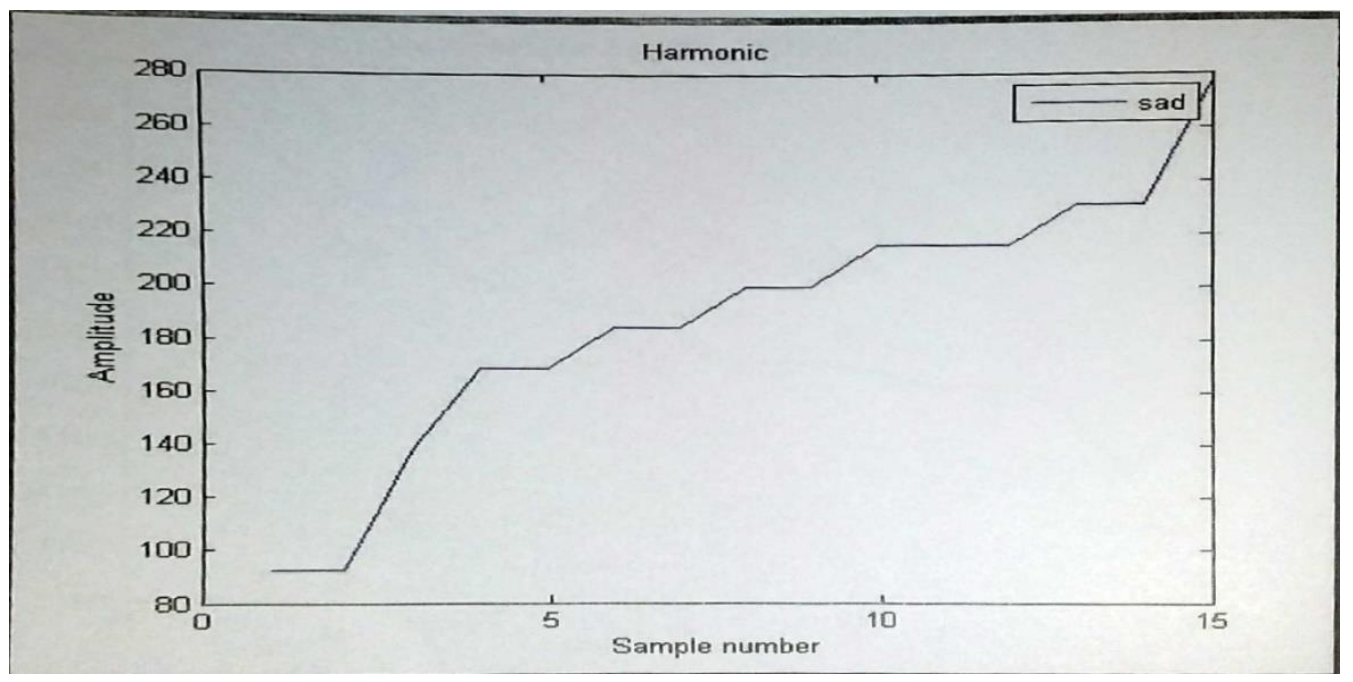

الشكل(12): قيمة التردد الأساسي(f0) للعينة

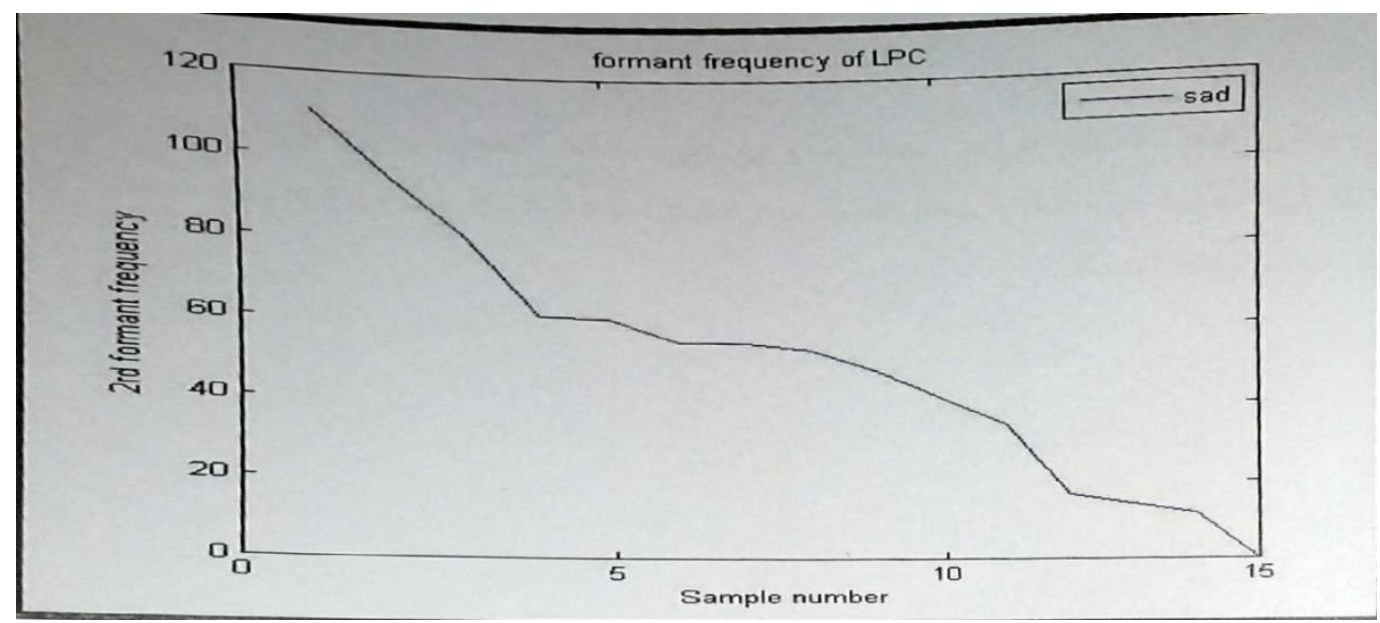

الثكل(13): قيمة التردد الرنلن(f2) للعينة 


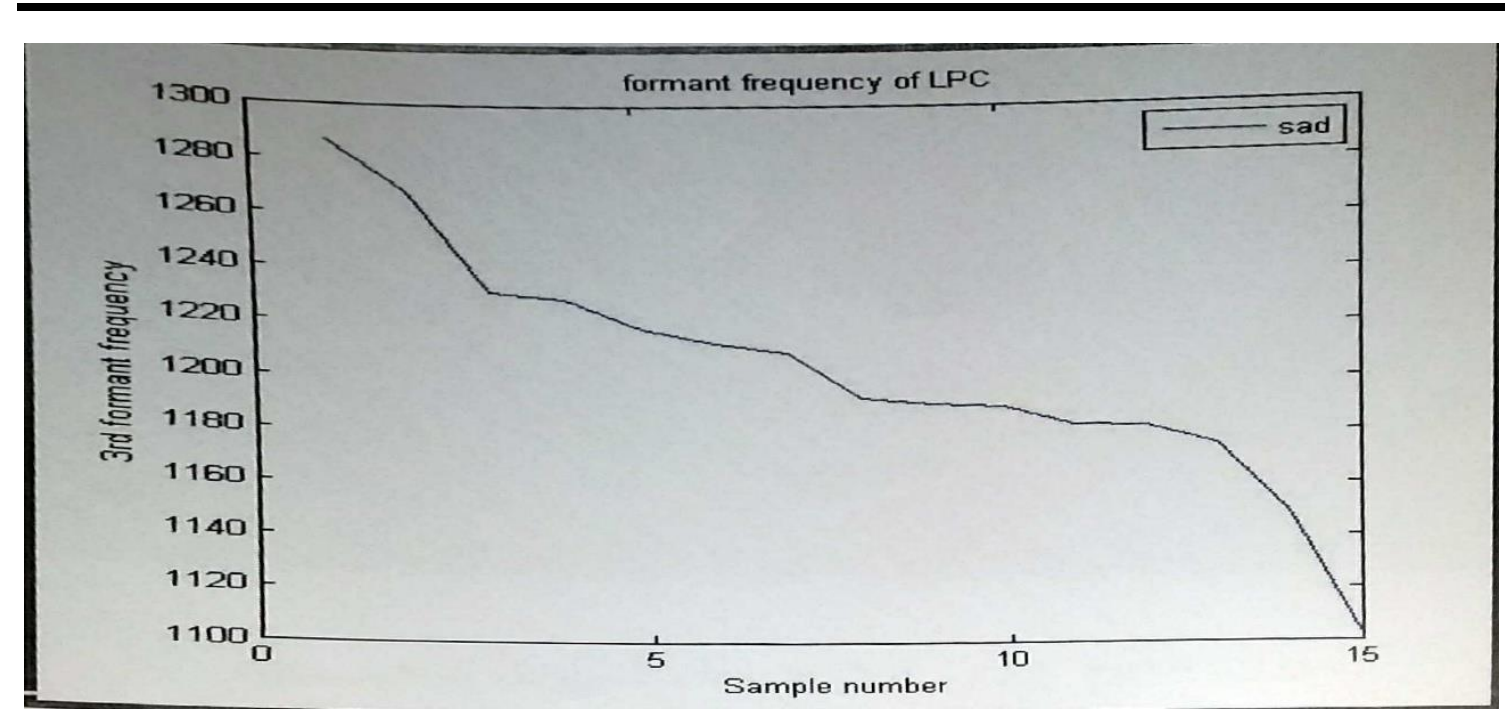

الثكل(14): قيمة التردد الرنين(f3) للعينة

وبعد إيجاد قيم تلك الخصائص للشخص المراد تحديد حالته المزاجية يتم إيجاد كل من معامل الارتباط والمسافة الإقليدية بين قيم تلك الخصائص والقيم المقابلة لها في المنحنيات السابقة التي تم الحصول عليها في مرحلة إعداد قاعدة البيانات، روالتي كانت تمثل أعلى ترابط وأقل مسافة إقليدية مع المنحني الذي يمثل حالة الحزن.

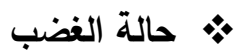

قرئ في هذا المثال ملف صوتي في حالة الغضب(angry.wav) وأجريت المعالجة الأولية عليه من إلغاء حالة الصمت والمعالجة بالنافذة ومن ثم يتم تقطع الإشارة إلى عدد من المقاطع طول كل مقطع 512كينة ليتم فيما بعد استخلاص الخواص منها والمتمثلة بالطاقة والتردد الأساسي والترددات الرنانة والأشكال من (15) إلى عنى (18) تمثل منحنيات تلك الخواص.

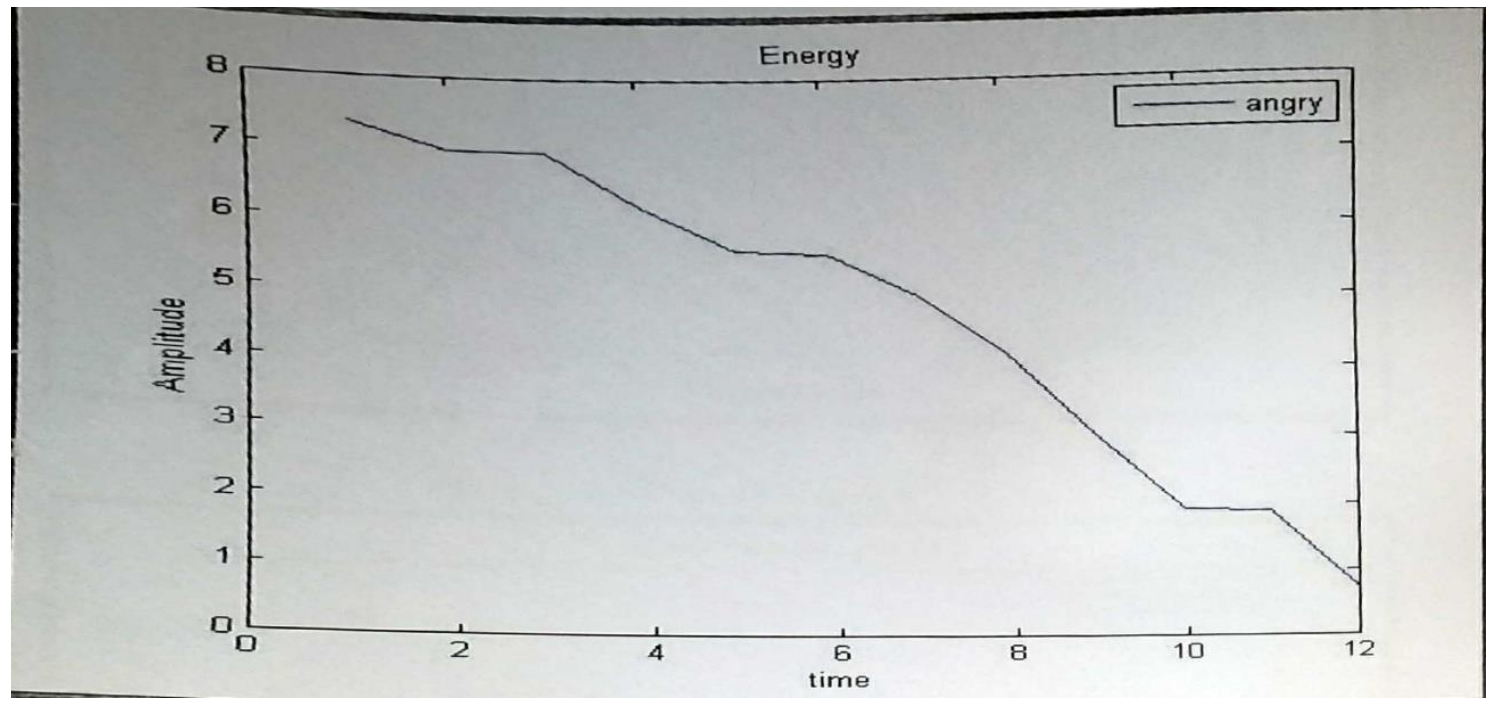

الثكل(15) قيمة الطاقة للعينة 


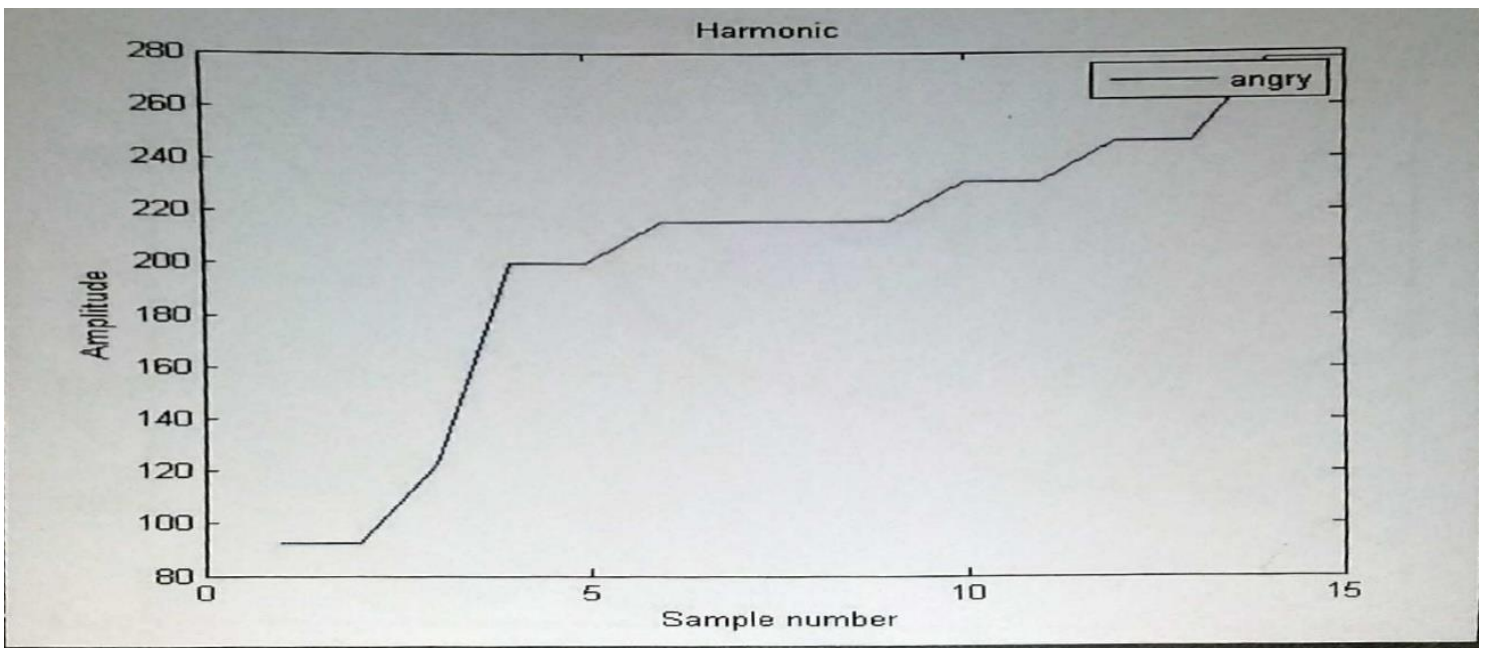

الثكل(16): قيمة التردد الأساسي(f0) للعينة

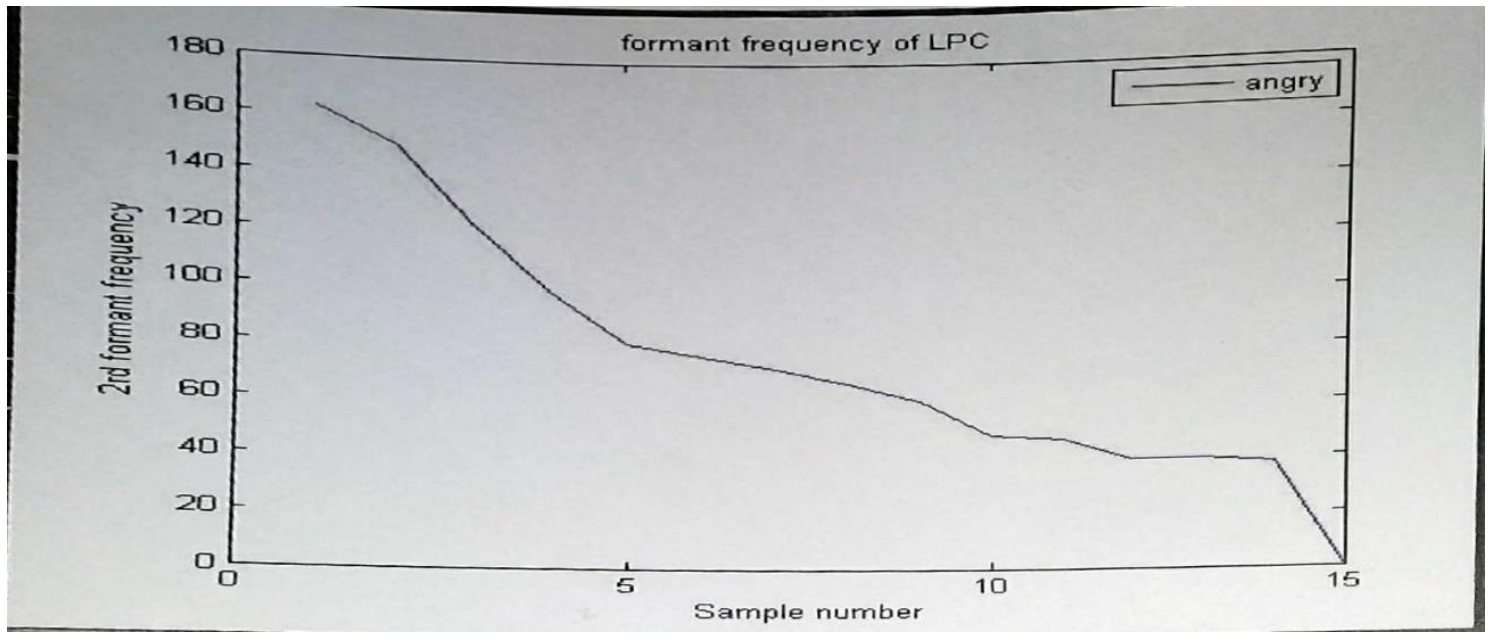

الثكل(17): قيمة التردد الرنان(f2) للعينة

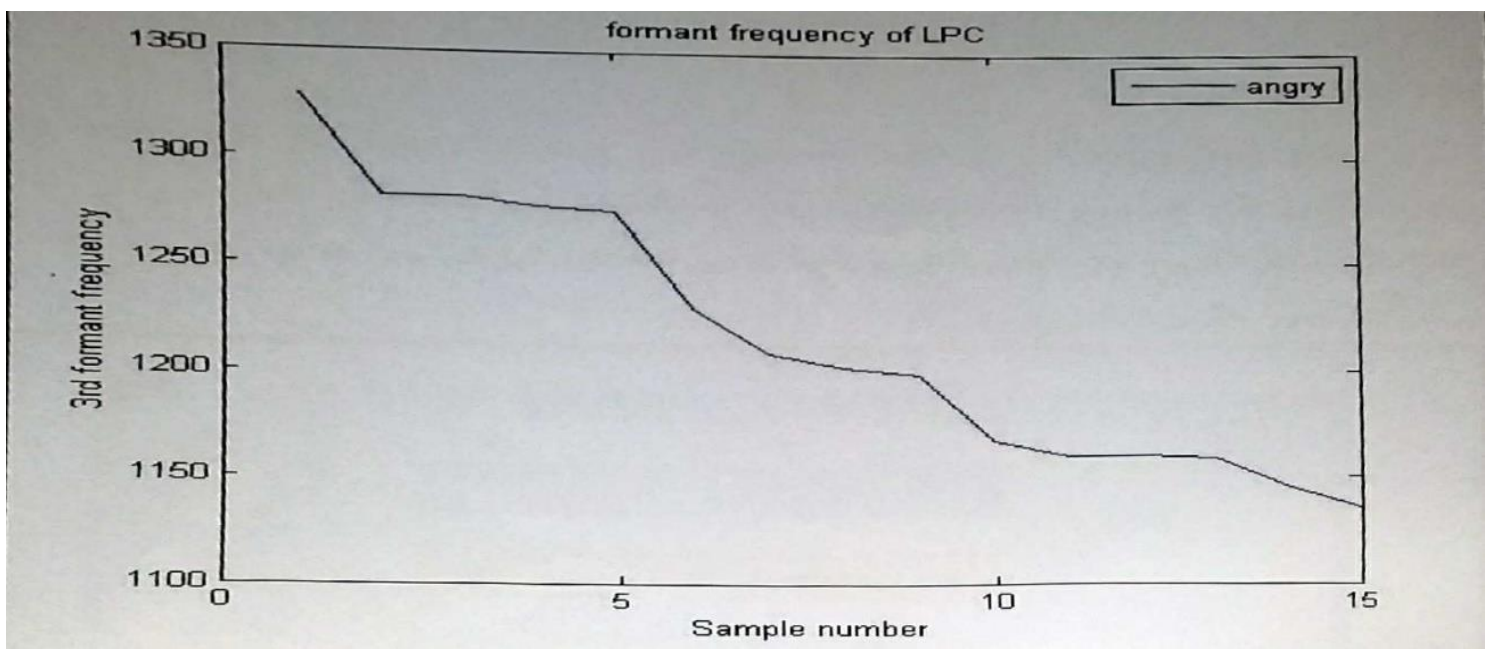

الثكل(18): قيمة التردد الرنان(f3) للعينة 
وبعد إيجاد قيم تلك الخصائص للثخص المراد تحديد حالته المزاجية يتم إيجاد كل من معامل الارتباط والمسافة

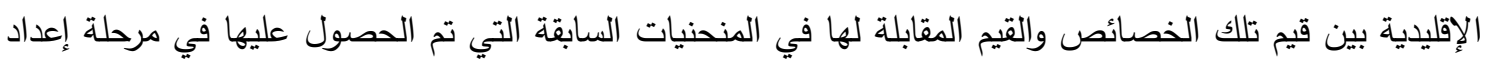
قاعدة البيانات، إذ أعطت أعلى ارتباط وأقل مسافة إقليدية مع المنحني الذي يمثل حالة الغضب. لإنب. 10. - 10

1. إن خواص اشارة الكلام المتمثلة بالطاقة(مجال الزمن)، التردد الأساسي، الترددات الرنانة(مجال التردد) أعطت نتائج جيدة بدقة تحديد الحالة المزاجية للمتكلم.

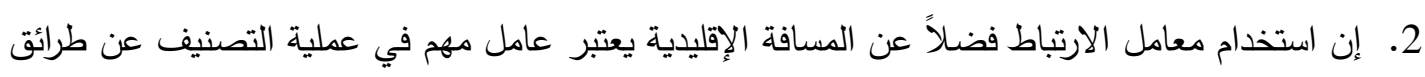
عملية إجراء المقارنة. 3. إن تقسيم إشارة الكلام إلى عدد من المقاطع بطول512عينة للمقطع الواحد واستخلاص الخصائص لكل مقطع أعطت نتائج جيدة عند اعتماد هذا الطول.

\section{1. الأعمال المستقبلية}

1. 1 إمكانية استخدام طرائق أخرى في التصنيف كالطرائق الذكائية.

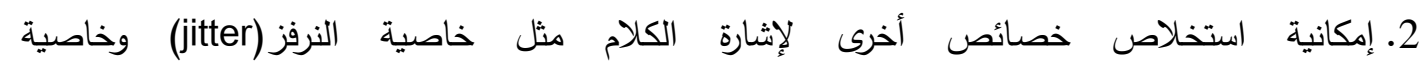
الوميض(shimmer) ودراسة تأثيرها على دقة التصنيف. 3. إمكانية اعتماد عدد أكبر من العينات في قاعدة البيانات ودراسة تأثيرها على عملية التصنيف داتصفيف. 


\section{المصادر}

[1] Kuhn Lisa Katharina, 2014,"emotion recognition in the human face and voice", Doctor thesis of Philosophy.

[2] S. D’Mello et. al, 2011,"Automatic Emotion Recognition from Speech", PhD Research Proposal (Eds.), ACII 2011, Part II, LNCS 6975, pp. 191199, (C) Springer-Verlag Berlin Heidelberg 2011.

[3] From Wikipedia, the free encyclopedia. https://en.wikipedia.org/wiki/Emotion_recognition

[4] Anagnostopoulos, Christos Nikolaos, et.al, 2015,"Features and classifiers for emotion recognition from speech: a survey from 2000 to 2011",Springer Science+Business Media Dordrecht, Volume 43, Issue 2, pp 155-177.

$$
\text { مبد النبي، هبه أدريس يونس، 2011، "تحديد الفئة العمرية للمتكلم باستخدام القيم المميزة"، بحث }
$$

[6] SchullerBjörn, et. al , 2002, "automatic emotion recognition by the speech signal", from SCI 2002, IIIS.

[7] Zbancioc Marius, Feraru Monica, 2012, "a study about the statistical parameters used in the emotion recognition", $11^{\text {th }}$ international conference on development and application systems.

[8] BhargavaaMayank., Polzehlb Tim, 2012, "Improving Automatic Emotion Recognition from speech using Rhythm and Temporal feature",In Proceedings of ICECIT-2012 Published by Elsevier.

[9] S.Li,et. al, 2014,"speech emotion recognition based on coiflet wavelet packet cepsetralcoefficients",part II, (C) Springer-Verlag Berlin Heidelberg 2014, CCIS 484, pp 436-443.

[10] Gluge,S., et. al, 2017,"emotion recognition from speech using representation learning in extreme learning machines", in proceeding of the $9^{\text {th }}$ international joint conference on computational intelligence, pp179-185, ISBN:978-989-758-274-5.

[11] Kerkeni,L.,et. al, 2018,"speech emotion recognition:methods and cases study",in proceedings of the $10^{\text {th }}$ international conference on agents and artificial intelligence(ICAART 2018), vol 2, p 175-182.

$$
\begin{aligned}
& \text { [12]قدو، سجى جاسم محمد، 2004،" كبس اشارة الكلام بواسطة استخلاص الخواص"، بحث } \\
& \text { ماجستير، علوم حاسوب، كلية علوم الحاسوب والرياضيات، جامعة الموصل. }
\end{aligned}
$$

[13] Pavol Partial, et.al,"impact of emotions on fundamental speech signal frequency", latest trends in information technology, ISBN: 978-1-61804134-0, p.409-414.

[14] Middleton, Gareth,2003,"pitch detection algorithms", openstaxCNXmodule:m11714. http://cnx.org/content/m11714/102/.

[15] MousaAllam, 2011,"speech segmentation in synthesized speech morphing using pitch shifting", The international Arab journal of information technology, vol.8, no.2, p221-226. 